\title{
Usefulness and efficiency of formalin-fixed paraffin-embedded specimens from laryngeal squamous cell carcinoma in HPV detection by IHC and PCR/DEIA
}

\author{
Kamal Morshed ${ }^{1}$, Malgorzata Polz-Dacewicz ${ }^{2}$, Marcin Szymański ${ }^{1}$, Agata Smoleń ${ }^{3}$ \\ ${ }^{1}$ Department of Otolaryngology Head and Neck Surgery, ${ }^{2}$ Department of Virology Medical, \\ ${ }^{3}$ Department of Mathematics and Biostatistics University of Lublin, Lublin, Poland.
}

\begin{abstract}
The use of formalin-fixed paraffin-embedded (FFPE) tissues for HPV DNA detection by PCR from biopsy materials is not entirely clear in retrospective studies. The aim of our study was to evaluate the usefulness and efficiency of FFPE tissues from laryngeal cancer (LSCC) in HPV detection by immunohistochemistry reaction (IHC) and PCR-DNA enzyme immunoassay method (PCR/DEIA) and to compare with HPV detection from DFT. HPV-DNA was amplified from 54 FFPE tissues from LSCC specimens by the short PCR fragment (SPF10) primer set using PCR/DNA method and monoclonal anti Human Papillomavirus antibodies in IHC. In the same patients 54 specimens were collected and immediately deep-frozen and stored at $\left(-70^{\circ} \mathrm{C}\right)$ to $\left(-80^{\circ} \mathrm{C}\right)$. All the FFPE and deep-frozen tissue (DFT) specimens were positive for $\beta$-globin amplification. HPV was detected by two methods (SPF10 PCR/DEIA and IHC) in $14(25.92 \%)$ out of 54 specimens from FFPE. Significant differences were found between the HPV detection using PCR/DEIA method and IHC method in FFPE tissues. The comparative analysis of the 54 samples after assuming PCR method in FFPE tissues showed accuracy of $92.6 \%$, sensitivity of $90.5 \%$ and specificity of $93.9 \%$. The FFPE tissues method has high sensitivity, specificity and accuracy when used to detect HPV DNA by PCR reaction and it is comparable to DFT results. DNA quality of FFPE samples is adequate and it can be used in HPV-DNA detection and in retrospective studies on LSCC.
\end{abstract}

Key words: FFPE, HPV, laryngeal cancer, SPF10, PCR/DEIA, IHC

\section{Introduction}

Papillomaviruses are members of the papovaviridae family. They are non-enveloped icosahedral DNA viruses, which infect humans as well as a variety of animals. The papillomavirus virion capsid consists of two proteins, L1 and L2. L1 is the major capsid protein and when expressed in eukaryotic expression systems it is able to self-assemble into virus-like particles (VLPs) [1,2]. Expression of L1 protein results in the self-assembly of virus-like particles, which have the size, shape and conformational epitopes of virion capsids. L1 is able to self-assemble into VLPs, morphologically and immunologically very similar to virions that are both necessary and sufficient for binding to the cell surface $[1,3]$. One hundred and eighteen papillo-

Correspondence: K. Morshed, Dept. of Otolaryngology Head and Neck Surgery, Medical University of Lublin, Jaczewskiego Str. 8, 20-954 Lublin, Poland; tel.: (+4881) 7244518, fax.: (+4881) 7244517, e-mail: kamal1@op.pl mavirus (PV) types have been completely described and approximately 120 additional isolates represent only partially characterized putative novel genotypes [4]. General or consensus PCR primers have been developed to detect a broad spectrum of HPV genotypes in a single PCR [5-7].

Extraordinary progress in molecular pathology has been made during the last 10 years, and molecular pathology techniques are moving rapidly from the research bench to routine utilization in diagnostic pathology. Several recent reports show that deepfrozen tissue (DFT) is a highly sensitive and specific material which can be used from clinical specimens to PCR detection of the HPV DNA. The majority of studies to date have used high quality DNA from frozen samples however these studies have been restricted due to the small number of samples in their collections. On the other hand, there is a huge resource of FFPE tissues specimens held in histopathology departments around the world. These samples provide an invaluable resource for studying the molecular basis of disease, making it possible to perform large retrospec- 
tive studies correlating molecular features with therapeutic response and clinical outcome.

The need for an effective archived material for the diagnosis of HPV infection in biopsy materials, for retrospective studies or to support histological findings, prompted us to evaluate the use of the $10 \%$ formaldehyde-fixed paraffin-embedded tissues.

The aim of our study was to evaluate the usefulness and efficiency of formalin-fixed paraffin-embedded (FFPE) tissues from LSCC in HPV detection by immunohistochemistry reaction (IHC) and PCR-DNA enzyme immunoassay method (PCR/DEIA) and to compare it with HPV detection from deep frozen tissues.

\section{Material and methods}

Tissue specimens were obtained from 54 consecutive patients with primary LSCC operated upon by the first author in the Department of Otolaryngology Head and Neck Surgery, Medical University of Lublin in the years 1999-2002. The group consisted of 45 males and 9 females, aged between 32 and 78 years (mean 57.7 years). Patients were treated surgically with or without postoperative radiotherapy depending on the clinical stage of the disease. The patients had neither previous radiotherapy nor chemotherapy.

In order to evaluate the study two specimens from the tumor were collected. For paraffin-embedded technique specimens were collected during a primary diagnostic microlaryngoscopy as a biopsy and they were fixed in $10 \%$ buffered paraformaldehyde and paraffin-embedded. Then they were referred to histopathological and virological lab for HPV DNA detection. The second specimens were collected from the excised tumors after total laryngectomy. The samples were immediately deep-frozen and stored at $\left(-70^{\circ} \mathrm{C}\right)$ to $\left(-80^{\circ} \mathrm{C}\right)$ for future analysis.

Immunohistochemical staining for HPV detection. Paraffinembedded tissue samples were sectioned serially at $4 \mu \mathrm{m}$ thickness. After de-paraffinization, sections were incubated with HPV monoclonal mouse antibodies and DAKO ${ }^{\circledR}$ Target Retrieval Solution for $40 \mathrm{~min}$. After washing with TBS samples were incubated with mouse monoclonal anti Human Papilloma virus antibodies Clone K1H8, DAKO (Produktionsvej 42, Glostrup, Denmark) (type: $6,11,16,18,31,33,42,51,52,56$, and 58) in dilution 1:50 with DAKO antibody Diluents for $60 \mathrm{~min}$ at room temperature. Samples were then incubated for $30 \mathrm{~min}$ with DAKO LASB ${ }^{\circledR}$ system at room temperature. Visualization of the reaction was acquired with DAB solution. After that, staining with Mayer's hematoxylin was performed. DAKO positive controls were used as a positive control. In negative controls original antibodies (DAKO) were not added. The detection of antibodies against in the nuclei of proliferating squamous cell carcinoma samples was regarded as a positive reaction.

\section{HPV detection using SPF10 PCR/DEIA method.}

DNA Extraction from paraffin sections. Five, $10 \mu \mathrm{m}$ sections of formalin-fixed, paraffin-embedded tissue were transferred to Eppendorf vials after cutting deep into the block. The microtome blade was changed after each case. DNA extraction from tissue specimens was done using standardization Genomic Mini test for DNA extraction kit (Genomic DNA Prep Plus A\&A Biotechnology Gdynia-Poland). After deparaffinization a tissue sample was transferred to consecutive Eppendorf vials and incubated at $50^{\circ} \mathrm{C}$ with $100 \mu \mathrm{l}$ Tris buffer, $50 \mu \mathrm{l}$ universal lyses solution LT and $20 \mu \mathrm{l}$ proteinase $\mathrm{K}$ and it was rotated for complete digest of the tissue sample. After incubation $150 \mu \mathrm{LT}$ buffer were added and it was centrifuged for 3 minutes at 10000-15000 rpm and washed twice with $98 \%$ ethanol. The precipitated DNA was centrifuged for 1 minute at 10000-15000 rpm and again washed with $70 \%$ ethanol. The DNA was precipitated and dried. Dried DNA extracts were incubated for 5 minutes at room temperature with $200 \mu$ Tris buffer (10 mM TRIS.HCI pH 8.5) which had a $75^{\circ} \mathrm{C}$ temperature and than it was centrifuged for 1 minute at $10000-15000 \mathrm{rpm}$. The obtained DNA extracts was stored at $-25^{\circ} \mathrm{C}$ for further analysis.

$\beta$-globin amplification. To analyze DNA quality PCR amplification of $\beta$-globin was performed in a separate reaction using primers PC04 and KM29 with fragment of $205 \mathrm{pb}$. The sequence of the set primer of PCO4 was d(5'-CAA CTT CAT CCA CGT TCA CC-3') and for KM29 was d(5'-GGT TGG CCA ATC TAC TCC CAG G-3').

HPV DNA detection. HPV DNA was amplified by the short PCR fragment (SPF10) human papillomavirus primer set (Labo Bio-medical Products B.V. - Holland). The SPF10 primers amplify a 65-bp fragment from the L1 region of the HPV genome as described by Kleter et al. [6]. The PCR products were analyzed by both 3\% agarose gel electrophoresis and HPV DNA enzyme immunoassay (DNA/DEIA). Each run was accompanied by quality control samples. During each PCR run, samples were tested, together with one negative control (distilled $\mathrm{H}_{2} \mathrm{O}$ ) and one positive control (Caski culture cell line). Amplification products were tested by probe hybridization in a microtiter plate assay to detect the presence of HPV DNA as described earlier by Kleter et al. [6]. The assay also included appropriate negative and positive controls.

Control samples. DNA from the Caski cell line was used as a positive PCR control to assess the success of the amplification. PCR reagents lacking DNA (Distilled $\mathrm{H}_{2} \mathrm{O}$ ) served in each PCR amplification as a negative control. Detection of sufficient quality DNA was performed by $\beta$-globin assay.

DNA extraction from frozen tissue. DNA extraction from deep frozen tissue specimens of the tumor and the HPV-DNA detection with nested-PCR method was done using standardized Genomic Mini test for DNA extraction (Genomic DNA Prep Plus A\&A Biotechnology Gdynia-Poland) and E6/E7 HPV-16 PCR test (DNA-GDANSK II s.c.-Poland). The amplified products were visualized through $2 \%$ agarose gel with ethidium bromide staining. DNA quality control was performed using the $\beta$-globin amplification with human $\beta$-globin primers KM29 and PC04. Caski culture cell line served as a positive control. The $\beta$-globin DNA was amplified in all cases with LSCC.

Control samples. DNA from the Caski cell line was used as a positive PCR control to assess the success of the amplification. PCR reagents lacking DNA (Distilled $\mathrm{H}_{2} \mathrm{O}$ ) served in each PCR amplification as a negative control.

Ethical issues. The study project was approved by the Local Institutional Review Board.

Statistical analysis. Sensitivity and specificity were assessed by $2 \times 2$ contingency tables; Chi-square exact test was performed in order to evaluate the percentage differences between the two assays. Kappa coefficient with $95 \%$ confidence limits was also performed to evaluate the correlation between paraffin-embedded specimens and deep-frozen tissue. Statistical significance was defined as $\mathrm{p}<0.05$.

\section{Results}

The keynote of this study regarding HPV DNA detection was a type of collected specimens for DNA extraction to evaluate the efficiency of paraffin-embedded tissue from LSCC in detection of HPV DNA compared to DFT. Both the histological diagnoses and the evaluation of the 
immunohistochemical reactions were carried out by two experienced pathologists in a blinded fashion. All the FFPE and DFT specimens were positive for $\beta$-globin amplification, the internal amplification control, and therefore were considered suitable for HPV analysis by $\mathrm{SPF}_{10}$ PCR/DEIA.

Human papillomavirus was detected by two methods ( $\mathrm{SPF}_{10}$ PCR/DEIA and IHC) in 14 (25.92\%) out of 54 specimens from FFPE tissues.

\section{Comparison of HPV detection from FFPE using SPF ${ }_{10}$ PCR/DEIA and IHC methods}

Human papillomavirus was detected in 21 (38.8\%) out of 54 paraffin-embedded specimens using $\mathrm{SPF}_{10}$ PCR/DEIA method and in $14(25.92 \%)$ out of 54 paraffin-embedded specimens using IHC method.

Seven samples from paraffin-embedded tissues were positive in HPV detection using $\mathrm{SPF}_{10}$ PCR/DEIA method and negative in HPV detection using IHC method with Mayer's hematoxylin staining but in these patients the deep-frozen specimens were also positive for HPV detection using $\mathrm{SPF}_{10}$ PCR/DEIA method. The positive results in HPV detection using $\mathrm{SPF}_{10}$ PCR/DEIA method and negative in HPV detection using IHC method resulted from differences in the number of HPV types detected by two methods. The IMH method detect 13 types of HPV and the $\mathrm{SPF}_{10}$ PCR/DEIA detect 25 types of HPV.

Characteristically, in all specimens with nuclear negative HPV detection in IHC test HPV was detected in the cytoplasm but it was regarded as negative. Significant differences were found between the HPV detection using PCR/DEIA method and IHC method from paraffin-embedded tissue $\left(\chi^{2}=4.00 ; \mathrm{p}=0.045\right)$.

\section{Comparison of HPV detection from FFPE and DFT using SPF ${ }_{10}$ PCR/DEIA method}

Using $\mathrm{SPF}_{10}$ PCR/DEIA method human papillomavirus was detected in 21 (38.8\%) out of 54 paraffinembedded specimens and in $21(38.8 \%)$ out of 54 deep-frozen tissues. It was the same patients.

Two samples of deep-frozen tissues were positive for HPV detection using SPF10 PCR/DEIA method and negative in paraffin-embedded tissue. This two samples were also negative for HPV using IHC method. No significant correlation was found between HPV detection using PCR/DEIA method from paraffin-embedded tissues and from deep-frozen tissues $\left(\chi^{2}=0.25 ; \mathrm{p}=0.61\right)$.

\section{Sensitivity and specificity of HPV detection from FFPE}

Overall, in comparison with deep-frozen tissue as the gold standard for PCR reaction, the paraffin-embedded tissue assay showed sensitivity of $90.5 \%$ (CI: 72.398.3 ) and specificity of $93.9 \%$ (CI: 81.6-98.9). The comparative analysis of the 54 samples after repeat testing of discordants showed accuracy of $92.6 \%$, $(\kappa($ Cohen's kappa $)=0.84 ; \mathrm{SE}=0.07 ; 95 \%$ CI: 0.71 $0.98)$.

\section{Discussion}

The goal is to elevate the quality of FFPE tissue obtained in clinical trials as the basis for discovering and validation of new biomarkers. Central to these efforts is the appreciation that tissue is no longer used only for microscopic interpretation of histopathology, but also for molecular assays of both nucleic acids (DNA), RNA and proteins.

Molecular techniques are rapidly gaining importance as adjuncts to histological tissue assessment. Since disease-related molecules harboring genetic as well as morphological disease characteristics are locked away in the vast collection of formalin-fixed paraffin-embedded tissues stored by the world's pathologists [8,9], it is crucial to evaluate the applicability of new molecular tools for routinely stored human FFPE tissues $[8,10]$. FFPE tissue samples have been collected throughout decades of routine histopathological examination and are thus the most widely available material in tissue archives around the world [8,9]. Many groups have found improved RNA recovery by disposing of the first sections of the block and using deeper sections for the isolation of RNA. Many laboratories routinely work with 20 -year-old material without any problem [11], and it is not unusual to encounter material that is older than 50 years. In fact, nucleic acids have been recovered from pathology museum specimens dating back to the early 20th century (Leeds) and to the 1918 influenza epidemic [12]. The studies were done on much older material delivered from extinct beings including early humanoids. The study material was not necessarily properly stored but found in archaeological excavations (Africa) or simply discovered in hardly accessible regions of Alps or Siberia. Interim approaches based on mtDNA differences between Neandertals and current humans, detection of male contamination through Y chromosomal sequences, and repeated sequencing from the same fossil to detect autosomal contamination allow initial large-scale sequencing of Neandertal genomes. For analyses of other fossil hominins, which may become possible in the future. Green et al. [13] suggest a similar 'boot-strap' approach in which interim approaches are applied until sufficient data for more definitive direct assays.

Different materials have been proposed to detect the presence of HPV in tissues. Most of researchers used the deep-frozen tissue as the gold standard of 
material to detect HPV DNA using a standardized PCR-based technique. Although frozen tissue is the gold standard for the isolation of biomolecules, the proposal of Medeiros et al. [14] for creation of tissue banks with storage at $-80^{\circ} \mathrm{C}$ is impractical for routine clinical care. Storage in mechanical freezers at $-80^{\circ} \mathrm{C}$ is inadequate to maintain RNA integrity for long periods [15]. Storage and retrieval in vapor-phase liquid nitrogen freezers are expensive and technically complicated. These approaches cannot be implemented without significant cost to community hospitals and clinics, where most specimens are obtained.

In this study we compared the usefulness and efficiency of paraffin-embedded specimens from LSCC in detecting the presence of HPV DNA with deep-frozen tissue obtained from the resectable tumor during tumor excision. We demonstrated that FFPE tissue has high sensitivity, specificity and accuracy in comparison with deep-frozen tissue for HPV detection in LSCC. These findings confirm and expand the results of studies of other authors for lung and cervical cancers [1618]. Farragher et al. [16] observed a remarkably high level of present cells in both frozen and FFPE samples. When they examined the overlap between fresh frozen and FFPE tissue expression data, they observed that despite the inevitable loss of information in FFPE tissue, both the specificity and the sensitivity were very high. The sensitivity was $71 \%$, indicating that the transcripts detected in frozen material could be detected in FFPE material and the specificity was $96 \%$ with the vast majority of the transcripts detected in FFPE also detected in frozen samples. These results were significantly higher than previously reported $[17,18]$ and provided confidence that reliable high quality data can be derived from FFPE tissue by the Lung Cancer research tool. Bibikova et al. [19] found that $90 \%$ of the genes detected in fresh frozen samples were detected in FFPE tissue; however, the gene expression proWles from FFPE did not correlate consistently present among technical replicates demonstrated similar and very low levels of noise in the data and equally high levels of correlation on both arrays.

Some authors in search of material to investigation which is simplified and easy in application on delivery applied HPV-DNA assays can use the same specimen as is used for cytological examination, which is an important logistic aspect of routine clinical testing. However, a scrape is only a small sample of the epithelium and sampling errors may influence cytology examination. Only a portion of cervical cell suspension is used for DNA isolation with only a fraction of the isolated DNA being used for specific DNA detection. Therefore, if a specimen contains only a limited number of HPV-DNA copies, sampling errors may produce inconsistencies even in a sensitive assay. Furthermore, the outcome of a HPV-DNA assay can vary depending on the menstrual cycle [20]. This not only has consequences for determining HPV-DNA presence or absence, but also could influence the accuracy of HPV detection, particularly when multiple HPV genotypes are present at different concentrations. Quint et al. [21] revealed that analysis of cervical scrapes as well as biopsy specimens from the same patient yielded comparable, but not identical HPV genotyping results. Thus, sampling errors should always be taken into account. Another study has compared biopsy to brush material of a tumor and margin for HPV detection in malignant laryngeal lesions. The results showed a positivity rate of $66.7 \%$ from biopsy material compared to $16.7 \%$ from brushings of the tumors [22].

The current study revealed that fixed in formalin paraffin-embedded specimens can be collected both for histological examinations and for HPV-DNA detection and these procedures can be performed using the same specimen, which is an important logistic aspect in routine clinical testing. However, the specimens taken from the larynx during the microlaryngoscopy are also small samples of the laryngeal infiltration and sampling errors may influence histological examination. The stability of the sample with $10 \%$ buffered paraformaldehyde during transport and storage is also important and give us the possibility of different investigations with nucleic acids for molecular diagnosis even after prolonged storage at ambient temperatures. The study revealed also that DNA quality of $10 \%$ buffered paraformaldehyde FFPE samples is adequate and it can be used for HPV-DNA detection and for retrospective researches. A minor limitation is the significant degree of degradation and chemical modification of the nucleic acids recovered from fixed tissues, but the sample collection with $10 \%$ buffered paraformaldehyde can reduce this limitation and we can control the quality of DNA using the $\beta$-globin test.

To conclude, FFPE tissues have a high sensitivity, specificity and accuracy in detecting HPV DNA infection by PCR reaction and it is comparable to DFT results. DNA quality of $10 \%$ buffered paraformaldehyde FFPE samples is adequate and it can be used for HPV-DNA detection and for retrospective researches even after prolonged storage at ambient temperatures.

\section{References}

[ 1] Kinrbauer R, Booy F, Cheng N, Lowy R, Schiller JT. Papillomavirus L1 major capsid protein self-assembles into viruslike particles that are highly immunogenic. Proc Natl Acad Sci USA. 1992;89:12180-12184.

[2] Rose RC, Bonnez W, Reichman RC, Garcea RL. Expression of human papillomavirus type $11 \mathrm{~L} 1$ protein in insect cells: in vivo and in vitro assembly of viruslike particles. $J$ Virol. 1993;67:1936-1944.

[3] Roden RB, Kirnbauer R, Jenson AB, Lowy DR, Schiller JT. Interaction of papillomaviruses with the cell surface. $J$ Virol. 1994;68:7260-7266. 
[4] de Villiers EM, Fauquet C, Broker TR, Bernard HU, zur Hausen H. Classification of papillomaviruses. Virology. 2004;324:17-27.

[5] Jacobs MV, Snijders PJ, van den Brule AJ, Helmerhorst TJ, Meijer CJ, Walboomers JM. A general primer GP5+/GP6(+)mediated PCR enzyme immunoassay method for rapid detection of 14 high-risk and 6 low-risk human papillomavirus genotypes in cervical crapings. J Clin Microbiol. 1997;35: 791-795.

[6] Kleter B, van Doorn LJ, ter Schegget J, et al. Novel shortfragment PCR assay for highly sensitive broad-spectrum detection of anogenital human papillomaviruses. $\mathrm{Am} \mathrm{J}$ Pathol. 1998;153:1731-1739.

[7] Kleter B, van Doorn LJ, Schrauwen L, et al. Development and clinical evaluation of a highly sensitive PCR-reverse hybridization line probe assay for detection and identification of anogenital human papillomavirus. J Clin Microbiol. 1999; 37:2508-2517.

[ 8] Srinivasan M, Sedmak D, Jewell S. Effect of fixatives and tissue processing on the content and integrity of nucleic acids. Am J Pathol. 2002;161:1961-1971.

[ 9] Specht K, Richter T, Müller U, Walch A, Werner M, Höfler H. Quantitative gene expression analysis in microdissected archival formalin-fixed and paraffin-embedded tumor tissue. Am J Pathol. 2001;158:419-429.

[10] Abrahamsen HN, Steiniche T, Nexo E, Hamilton-Dutoit SJ, Sorensen BS. Towards quantitative mRNA analysis in paraffin-embedded tissues using real-time reverse transcriptasepolymerase chain reaction: a methodological study on lymph nodes from melanoma patients. J Mol Diagn. 2003;5:34-41.

[11] Cronin M, Pho M, Dutta D, et al. Measurement of gene expression in archival paraffin-embedded tissues: development and performance of a 92-gene reverse transcriptase-polymerase chain reaction assay. Am J Pathol. 2004;164:35-42.

[12] Taubenberger JK, Reid AH, Krafft AE, Bijwaard KE, Fanning TG. Initial genetic characterization of the 1918 "Spanish" influenza virus. Science. 1997;275:1793-1796.

[13] Green RE, Briggs AW, Krause J, et al. The Neandertal genome and ancient DNA authenticity. EMBO J. 2009;28: 2494-2502.
[14] Medeiros F, Rigl CT, Anderson GG, Becker SH, Halling KC. Tissue handling for genome-wide expression analysis: a review of the issues, evidence and opportunities. Arch Pathol Lab Med. 2007;131:1805-1816.

[15] Fricain JC, Rouais F, Dupuy B. A two-step embedding process for better preservation of soft tissue surrounding coral implants. J Biomed Mater Res. 1996;33:23-27.

[16] Farragher SM, Tanney A, Kennedy RD, Paul Harkin D. RNA expression analysis from formalin fixed paraffin embedded tissues. Histochem Cell Biol. 2008;130:435-445.

[17] Linton KM, Hey Y, Saunders E, et al. Acquisition of biologically relevant gene expression data by Affymetrix microarray analysis of archival -fixed parafin-embedded tumours. $\mathrm{Br} J$ Cancer. 2008;98:1403-1414.

[18] Scicchitano MS, Dalmas DA, Bertiaux MA, et al. Preliminary comparison of quantity, quality, and microarray erformance of RNA extracted from -Wxed, parafin-embedded, and unfixed frozen tissue samples. J Histochem Cytochem. 2006; 54:1229-1237.

[19] Bibikova M, Talantov D, Chudin E, et all. Quantitative gene expression profiling in formalin-fixed, paraffin-embedded tissues using universal bead arrays. Am J Pathol. 2004;165: 1799-1807.

[20] van Ham MA, Melchers WJ, Hanselaar AG, Bekkers RL, Boonstra H, Massuger LF. Fluctuations in prevalence of cervical human papillomavirus in women frequently sampled during a single menstrual cycle. Br J Cancer. 2002;87:373376.

[21] Quint WG, Scholte G, van Doorn LJ, Kleter B, Smits PH, Lindeman J. Comparative analysis of human papillomavirus infections in cervical scrapes and biopsy specimens by general SPF(10) PCR and HPV genotyping. J Pathol. 2001;194:5158.

[22] Vowles RH, Myint S, Croft CB. The incidence and detection of HPV in the upper aerodigestive tract using brush and biopsy techniques. J Laryngol Otol. 1997;111:215-217.

Submitted: 23 December, 2009 Accepted after reviews: 21 March, 2010 